\title{
An Intelligent Matching System for the Products of Small Business/Manufactures with the Celebrities
}

\author{
Junho Jeong ${ }^{1}$, Yunsik Son ${ }^{2}$, Seokhoon $\mathrm{Ko}^{1}$ and Seman $\mathrm{Oh}^{1}$ \\ ${ }^{1}$ Dept. of Computer Engineering, Dongguk University, Seoul, Korea \\ ${ }^{2}$ Department of Brain and Cognitive Engineering, Korea University, Seoul, Korea
}

\begin{abstract}
With the development of ICT, e-business have brought consumers opportunities to choose a wider range of products to purchase. However, these opportunities made it difficult for them to make a choice. Under this circumstance, product recommendation systems have arisen, helping consumers with purchase decision. In this paper, an intelligent matching system is proposed to connect small business/manufactures with celebrities. Previous matching/recommendation systems using narrowing approach based on limited information or using widening approach based on users' information. But these approaches have many restricts. The proposed matching system produces suitable celebrity candidate set to advertise target product of small business/manufactures based on relationship graph which can compare the similarity of each celebrity.
\end{abstract}

\section{Introduction}

Recently, there are various recommendation systems for matching products between celebrities to increasing promotion effects exist. The goal of these systems is increasing sales volume by the selecting suitable celebrities as an advertisement model. But, the existing systems did not work effectively, because its matching methodologies have simple approaches and using few features to matching the target product with celebrities.

This paper proposes the new method to improve promotion effect through advertising by suitable matched celebrities for the given product with using star relationship graph. The proposed method collets the information of the celebrities automatically on the web, and generating the star relationship graph as the relationship/similarity of each celebrities using $\mathrm{KNN}$ distance with hierarchical random graph model.

\section{Related studies}

\subsection{Recommendation system}

With the development of ICT, e-business have brought consumers opportunities to choose a wider range of products to purchase. However, these opportunities made it difficult for them to make a choice. Under this circumstance, product recommendation systems have arisen, helping consumers with purchase decision. In recent years, rarely can we find online stores without this system which deals with various kinds of products.
As an information filtering system, the recommendation system predicts the preference or estimation of the users for the recommended item. The recommendation system is largely fall into two categories: context based filtering $[1,2]$ and collaboration filtering [3].

Table 1. Categories of recommendation system

\begin{tabular}{|c|c|}
\hline Category & \multicolumn{1}{c|}{ Contents } \\
\hline \multirow{1}{*}{$\begin{array}{c}\text { Context based } \\
\text { Filtering }\end{array}$} & $\begin{array}{c}\text { - using the information of user } \\
\text { or product's contents } \\
\text { - using the techniques of the } \\
\text { natural language processing } \\
\text { or information searching } \\
\text { - depend on product's } \\
\text { description (metadata) } \\
\text { - narrow recommendation range } \\
\text { - needed small size information }\end{array}$ \\
\hline Collaboration & $\begin{array}{l}\text { - using the estimation of users } \\
\text { - using the similarity of the } \\
\text { products }\end{array}$ \\
& $\begin{array}{l}\text { - using many users' estimation } \\
\text { information }\end{array}$ \\
& $\begin{array}{l}\text { - wide recommendation range } \\
\text { needed big size information } \\
\text { - divide by user based method } \\
\text { and item based method }\end{array}$ \\
\hline
\end{tabular}

Table 1 shows characteristics of the two kind of the recommendation system.

These recommendation systems used on various fields as product sales (Amazon, Netflix), news, video 
recommendation (Google, Youtube, Yahoo, Naver), social recommendation (Facebook).

\subsection{Hierarchical random graph}

A hierarchical random graph [4] is a random graph whose definition encodes some information about the expected community structure of the graph. The definition is given in terms of a dendrogram which gives information about the probability that any two nodes in the graph are connected. Each node has probabilities associated with other nodes, such that the probability for a node higher up the graph is strictly less than a node higher down the graph. In hierarchical random graph, any two vertices are joined by an edge with a probability corresponding to their lowest common ancestor in the dendrogram.

Because of the inequalities satisfied by the probabilities, this will naturally generate a graph with community structure (i.e. clusters of mutually connected vertices with a few connections to vertices in other clusters) and it called 'hierarchical' because, by the nature of the dendrogram, also it can describe the communities of communities, communities of communities of communities etc. Figure 1 shows example of data representation using the hierarchical random graph.

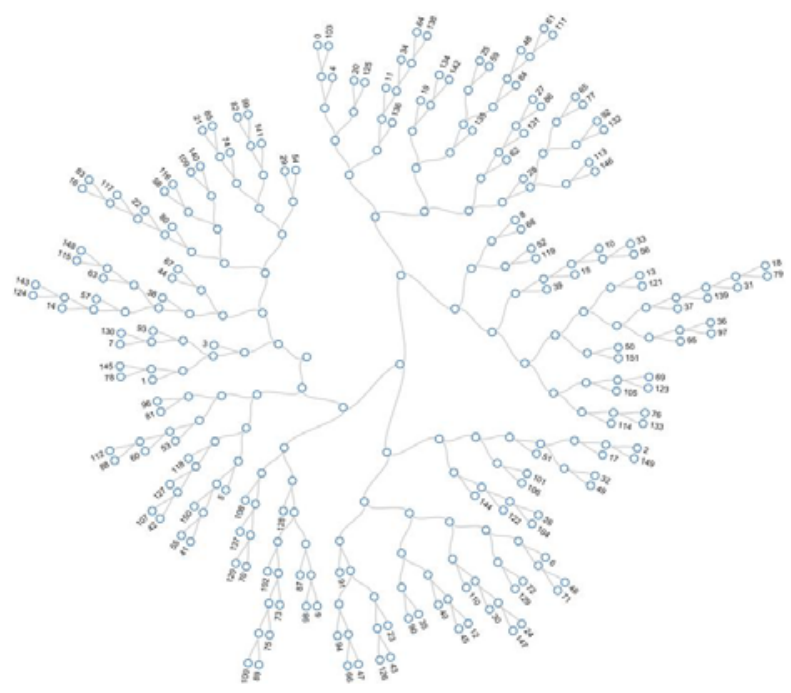

Figure 1. Visualization example of the comunity information represented by hierarchical rendom graph

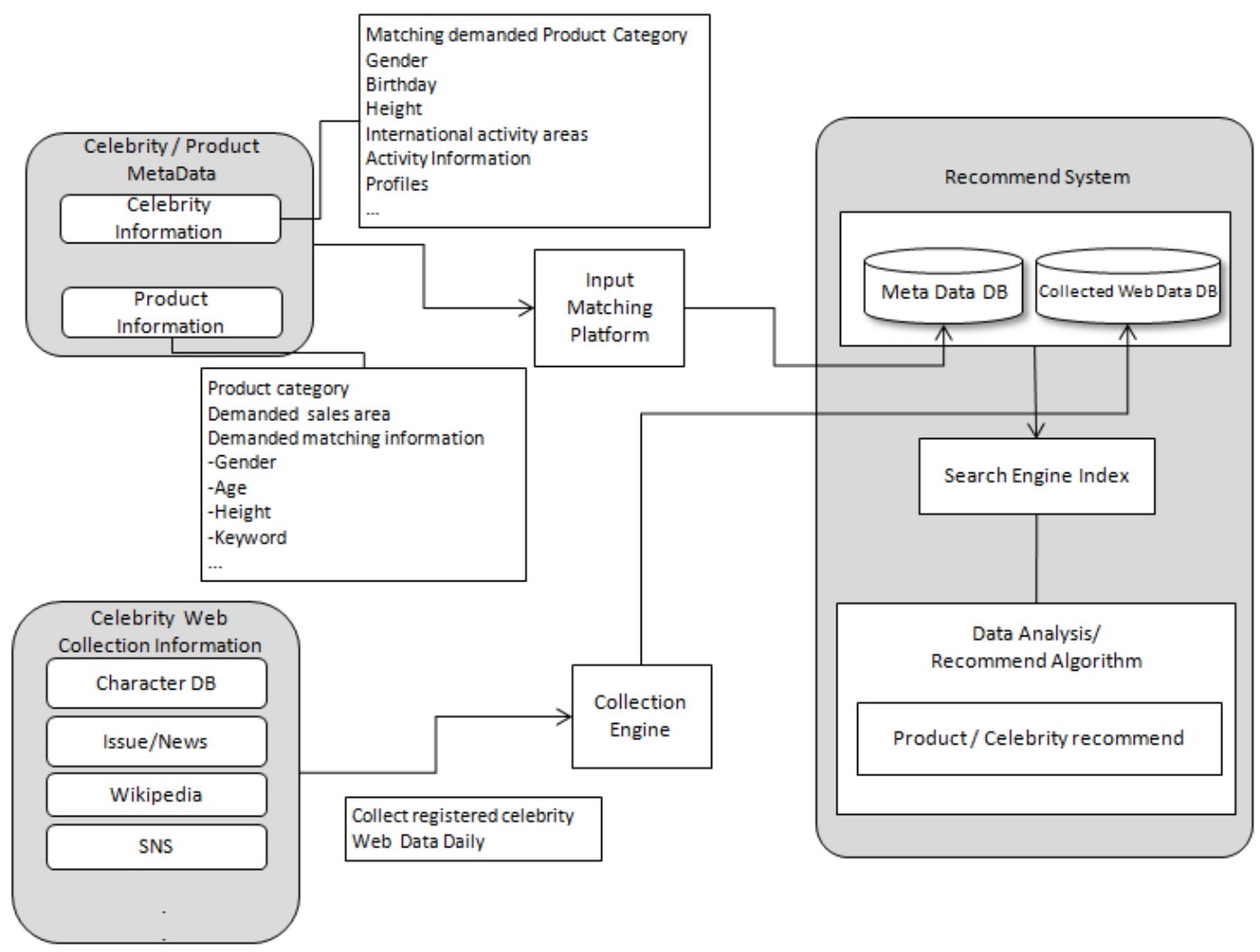

Figure 2. System structure of the intelligent matching system 


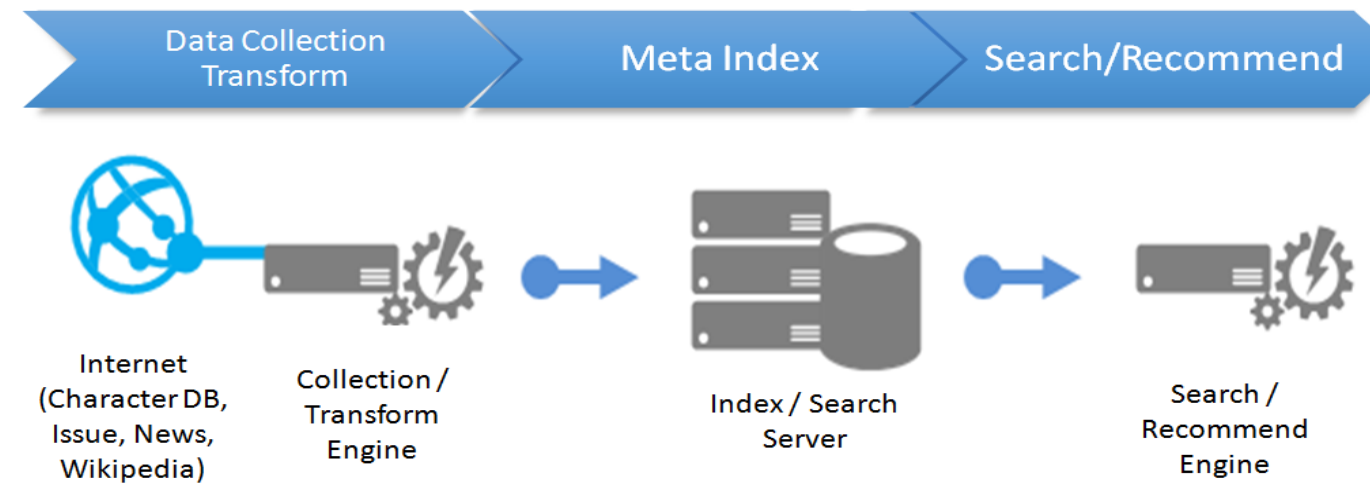

Figure 3. Data collection process for constructing star infromation

\section{Proposed business matching system}

Recommendation system is part of information filtering system. It is prediction and evaluation system about recommended item to consumer. Recommendation system depends on consumer, consumer's comments, natural-language processing using product's name, information retrieval and explanation of product. Therefore, the recommendation system can implement two methods. First is content-based filtering method. It recommends with small information but it has narrow recommendation range. Second is collaborative filtering. It uses consumer's evaluation to calculate similarity measure but it needs a large of information. Proposed system is a hybrid form [5] that takes advantage of the two implementations.

\subsection{Matching system model}

The proposed recommendation algorithm based on weight extracts effective matching result using small information. It is compatible recommendation system in the initial operating system. Figure 2 is a block diagram of a system like that proposed. Each system was constructed in modular function to handle the feedback type because of flexible data and matching result.

The system builds and index collected information to the database. The information is collected in two ways. One way is to collect from internet such as Character DB about celebrities, Issue/News, Wikipedia, and Social Network Services by Collection Engine. And other ways are to obtain Celebrities/Product meta data that made by manager of celebrity or product provider. The system recommends suitable celebrities for products by analyzing the index information to match the relationship between the product and the celebrities.

\subsection{Data collection method}

Data collection process gathering news information published on the web in order to collect information on the operating system constitutes the initial organization of the data as shown in Figure 3 shown collection steps.
An intelligent recommend system is required, such as Figure 2 is a meta data search engine as data collection/indexing/search due to the context based filtering. Celebrity metadata that is collected through the Web includes unstructured text data in addition to the formal data. Therefore, indexing engine is to generate a metadata index information by morphological analysis.

Typically, the default metadata of celebrity is managed directly by the himself or agency manager. However, there are practical limits to ensure immediate and effectiveness of data entry. Since Web collection engine to collect and classify the data in the various channels such as Character DB about celebrities, Issue/News, Wikipedia, and Social Network Services. The information is the metadata of the matching system provides the system operator and celebrities agency. As a result, by using this information it is able to be configured to increase the accuracy and probability of matching of the data for the matching system.

\subsection{Star relationship graph based matching model}

Proposed graph based matching model is using 2 kind of graph; first is PG (Product Graph) and next is SRG (Star Relationship Graph).

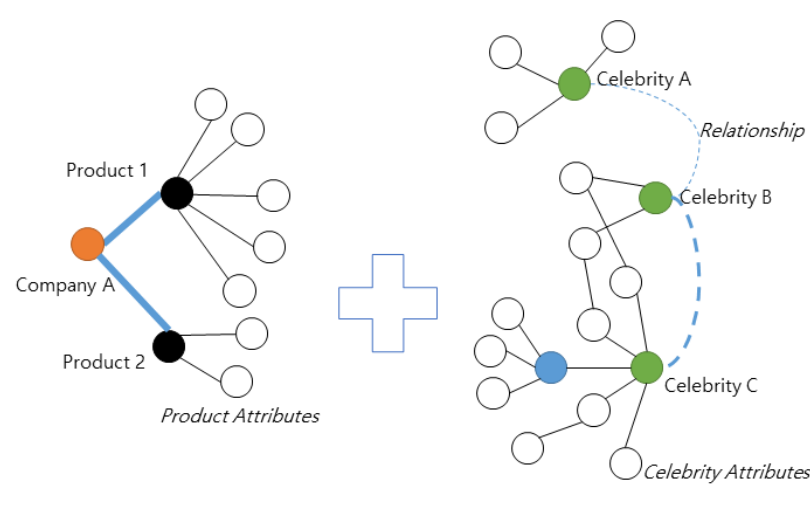

PG: Product Graph

SRG: Star Relationship Graph

Figure 4. Graph models for macthing system; Product graph \& star relationship graph

PG is constructed by attributes of the company's target products and SRG is combined by collected 
attributes by data collection process. Figure 4 shows the overall graph model. SRG based matching model as shown in Figure 5 was constructed celebrity as a model for improving the performance of a weight based algorithm, and others associated with the hierarchical graph.
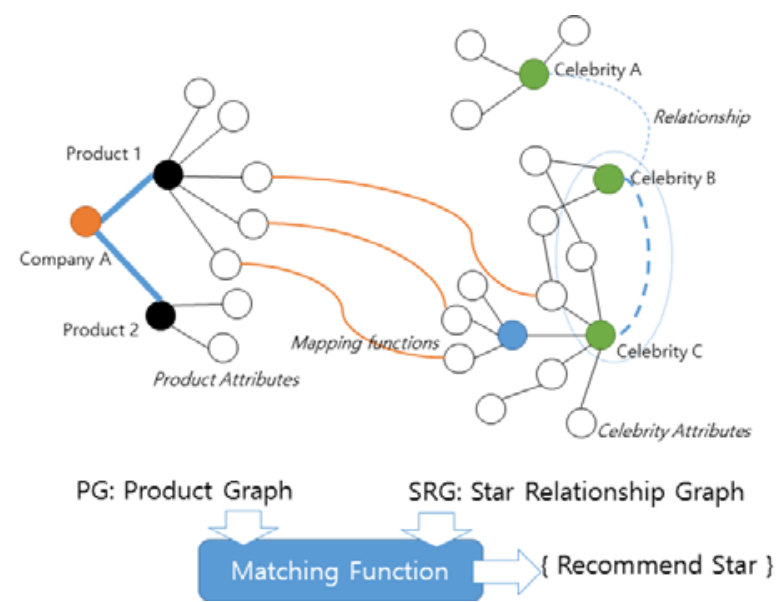

Figure 5. SRG besed matching model

It suggests a recommendation of celebrity group. It has two type of graph. PG (Product Graph) is made up classify product, prices, features, sales target and sales area node. SRG is made up celebrity of age, gender, active period, active state and the existing advertising items node. It generates matching result with graph mapping.
Distribute Form

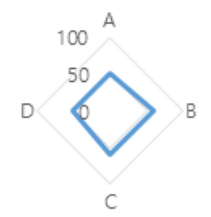

Awareness Form

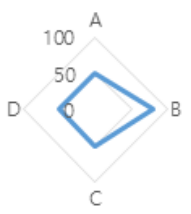

Affirmative Form

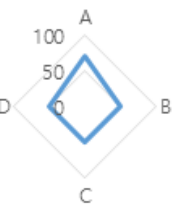

Commodity Form

$D$

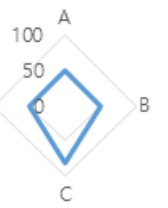

Issue Form

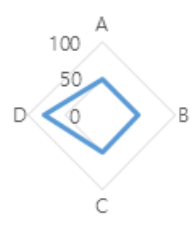

Figure 6. Specialized example forms using proposed metric

As Fig. 6, each celebrity is estimated by 4 metrics; Degree of Affirmative, Degree of Awareness, Degree of Commodity and Degree of Issue. Firstly, degree of affirmative is calculated by exposure rate of broadcasting, internet and number of SNS follower with posting. And next, degree of awareness is estimated by number of personal store and number of awards during one year. Degree of commodity is calculated using number of advertisement during one year, advertisement for competitors during one year, and estimated cost/profit. Finally, degree of issue is estimated by number of news article during one year and activated programs; TV, radio, films, and etc.

SRG generated using hierarchical random graph with KNN distance based on estimated celebrity information. Thus, each node of SRG can be find a similar node set and estimate the nearest with similar nodes.

Table 2 shows the matching process using the SRG and $\mathrm{PG}$.

Table 2. Matching process using SRG

1. Configure the celebrity set SRG graph using the information related to celebrities

2. SRG represents celebrity relationships depending on the property with each of the celebrities

3. Represent the information of the product in PG: Product Graph

4. Selecting the highest matching rate celebrity of the SRG and PG according to the pre-defined matching function,

5.Extract the relevance celebrities linked to selected celebrities from SRG

6. Send expected to get higher matching rate celebrity with product

\section{Conclusion and further research}

In this paper, the matching system based on relationship graph model was proposed. Proposed method designed to improve promotion effect through advertising by recommend suitable matched celebrities for target product using the relationship graph which represents the properties of celebrities and their similarity.

In the future, a process to evaluate the implemented matching system is necessary. And study to be elaborated the matching system how to reflect the preference and matching rate score is needed.

\section{Acknowledgements}

This work was supported by the Technology Innovation Program (10048990, Development of business matching platform and collaboration product with celebrity and Korean stars for the domestic and overseas business activation of small business and manufactures) funded by the Ministry of Trade, industry \& Energy (MI, Korea)

\section{References}


1. A. Ansari, S. Essegaier, R. Kohli. Internet Recommendations Systems, J. Mar. R. 37, 363 (2000)

2. G. Adomavicius, T. Alexander. oward the next generation of recommender systems: A survey of the state-of-the-art and possible extensions, IEEE $\mathrm{T}$. Know. Data Eng. 17, 734 (2005)

3. M. Balabanović, Y. Shoham. Fab: content-based, collaborative recommendation, Comm. ACM, 40, 66 (1997)

4. A. Clauset, C. Moore, M. E. Newman. Hierarchical structure and the prediction of missing links in networks, Nature 453, 98 (2008)

5. S. Debnath, N. Ganguly, P. Mitra. Feature weighting in content based recommendation system using social network analysis, 17th int. Conf. WWW, 1041 (2008)

6. R. Burke. Hybrid Recommender Systems: Survey and Experiments, User Mod. User-Adapt. Int. 12, 331 (2002)

7. Y. W. Chen. A collaborative filtering recommendation algorithm based on contents' genome, IET Int. Conf. Infor. Sci. Cont. Eng. 1 (2012) 